\title{
Results of Osteochondral Autologous Transplantation in the Knee
}

\author{
Sandra Muller ${ }^{*}, 1$, Roelf S. Breederveld ${ }^{2}$ and Wim E. Tuinebreijer ${ }^{3}$ \\ ${ }^{1}$ Department of Surgery, VU University Medical Center, Boelelaan 1117, $1081 \mathrm{HV}$ Amsterdam, The Netherlands \\ ${ }^{2}$ Department of Surgery, Red Cross Hospital, Vondellaan 13, 1942 LE Beverwijk, The Netherlands \\ ${ }^{3}$ Dutch Burns Foundation, P.O. Box 1015, 1940 EA Beverwijk, The Netherlands
}

\begin{abstract}
Repair of full thickness defects of articular cartilage in the knee is difficult but important to prevent progression to osteoarthritis. The purpose of this retrospective study was to evaluate the clinical results of Osteochondral Autograft Transplant System (OATS) treatment for articular defects of the knee.

Between 1999 and 2005, 15 knees (14 patients) were treated by the OATS technique. Age ranged from 27 to 52 years. Cartilage defects were up to $3.75 \mathrm{~cm}^{2}$. The mean follow-up was 42 months. Knee function was assessed by the Lysholmscore and International Knee Documentation Committee (IKDC) Subjective Knee Form. Six patients scored good or excellent. No patient had knee instability. Twelve of 13 patients returned to sports at an intermediate or high level. The subjective assessment score (0-10) changed from 4.7 before operation to 7.2 afterward $(\mathrm{P}=0.007)$. The OATS-technique resulted in a decrease in symptoms in patients with localized articular cartilage defects. We consider the OATS technique to be an appropriate treatment for cartilage defects to prevent progression of symptoms.
\end{abstract}

Keywords: Osteochondral autologous transplantation, articular cartilage, knee injuries, mosaicplasty.

\section{INTRODUCTION}

Articular cartilage is critical for proper joint function. It is vulnerable to traumatic injury and has no capacity to regenerate. Articular cartilage defects are caused by trauma, chronic instability, degeneration or osteochondritis dissecans (OCD). OCD is a condition in which a fragment of bone in the joint is deprived of blood and separates from the rest of the bone. Untreated cartilage defects may progress to osteoarthritis and correlate with pain and joint dysfunction.

There are various techniques to treat osteochondral defects, including abrasion, drilling, microfracture, chondrocyte transplantation and osteochondral autografts.

Abrasion involves debriding the articular defect to the bony surface. Thereafter the defect fills itself with a fibrin clot, which after 4-6 months results in fibrocartilaginous tissue. Another therapeutic option to create a fibrin clot is to make microfractures or drill the subchondral bone at the site of the defect $[1,2]$. However, fibrocartilaginous tissue is more fragile than normal hyaline cartilage [3]. There has been a recent increase of interest in more reliable and lasting cartilage repair techniques, where the defect is filled with hyaline cartilage, such as autologous chondrocyte implantation or autologous osteochondral transplantation [4, 5]. The latter technique is also called mosaicplasty or the Osteochondral Autograft Transplant System (OATS), and it achieves a congruency of the articular surface in the loadbearing zone of the femoral condyle [1,2]. An osteochondral cylinder of a non-weight-bearing part of the femur condyle is

*Address correspondence to this author at the Department of Surgery, VU University Medical Center, Boelelaan 1117, 1081 HV Amsterdam, The Netherlands; Tel: +31619179339; E-mail: sandramullerr@hotmail.com harvested and transplanted in the defective portion of a weight-bearing part of the femur condyle. Depending on the size of the defect, additional donor plugs can be placed next to each other to fill the defect. This technique can be done by arthroscopy, but larger lesions frequently need arthrotomy. Gudas et al. found good results with the OATS technique in $86-90 \%$ of patients after a follow-up period of three years [1, 2].

The OATS technique has been performed in our hospital since 1999. The purpose of this study was to evaluate the clinical results of patients with the OATS technique.

\section{MATERIALS AND METHODOLOGY}

This study investigated the functional outcome of 14 patients (15 knees) operated on using the OATS technique between 1999 and 2005 (Table 1). These patients had a local articular cartilage defect of up to $4 \mathrm{~cm}^{2}\left(1.5-3.75 \mathrm{~cm}^{2}\right)$ in the weight-bearing part of the medial $(\mathrm{N}=14)$ or lateral femur condyle. The defects were classified by grade III/IV according to the Outerbridge classification [6] and were diagnosed by MRI and arthroscopy. Lesions larger than 4 $\mathrm{cm}^{2}$ or patients with serious gonartrosis were excluded from the study. One woman was operated bilaterally. Four patients had their anterior cruciate ligament (ACL) reconstructed during the same operation. Mean age was 41 years and all patients were competitive or well-trained athletes.

During the first diagnostic arthroscopy, concomitant procedures were performed in six patients and included drilling $(\mathrm{N}=1)$, partial meniscectomy $(\mathrm{N}=3)$, ACL reconstruction $(\mathrm{N}=1)$ or partial meniscectomy and $\mathrm{ACL}$ reconstruction $(\mathrm{N}=1)$.

Patients were hospitalized for 4 days. Within two weeks, partial weight bearing of up to 15-30 kilograms was 
permitted, and after 4 to 6 weeks patients progressed to full weight bearing. Patients were allowed to return to work after 6 to 8 weeks and to play sports after 6 months.

Table 1. Patient Characteristics

\begin{tabular}{|l|c|}
\hline Sex & 2 female, 12 male \\
\hline Age in years & Mean $41.1, \mathrm{SD}=7.6$, min. 27, max. 52 \\
\hline Hospital stay in days & Mean $4.25, \mathrm{SD}=1.1$, min. 3, max. 6 \\
\hline Trauma in patients history & 10 patients positive \\
\hline Localisation defect knee & 14 medial, 1 lateral \\
\hline
\end{tabular}

The mean outpatient control period was 6.3 months (min. 2 and max. 15 months). For study purposes we invited all patients for a physical re-examination and a radiograph. Nine to 77 months after the operation 13 patients (14 knees) responded and two patients were lost to follow-up. To score the function of the knee the Knee Ligament Standard Evaluation Form and the Knee Examination Form (KEF) (IKDC-scoring system) and the Lysholm-score were used $[7,8]$. A maximum Lysholm-score reflects optimal function of the knee without complaints. Scores are assigned to four categories: Excellent (100-91), good (90-82), fair (81-62) and poor (61-0). The Subjective Knee Score Questionnaire (SKSQ) has been used to score subjective complaints. The radiographs of the knee pre- and postoperatively were scored by a radiologist blinded for patient data using the IKDC technique for medial, lateral, anterior, posterior and patellarfemoral joint space narrowing.

A statistical examination was performed with SPSS version 15.0 (SPSS, Chicago, Illinois). Means, medians, interquartile ranges and standard deviations were calculated. The Wilcoxon signed rank test was used for comparing paired data.

Procedures were performed by the same surgeon under spinal or general anaesthesia with a tourniquet-controlled bloodless field. No antibiotics were administered perioperatively. The knee joint was opened parapatellar. Remnants of residual cartilage and calcified layers of subchondral bone were removed from the defect. The size of the defect was measured using a $5-10 \mathrm{~mm}$ stamp. The donor site was at a non-weight-bearing part of the femur condyle, such as the lateral rim of the femur condyle above the sulcus terminals or at the superiolateral site of the inter-articular notch. Perpendicular to the articular surface a tubular chisel harvested one or more plugs of cartilage and subchondral bone of $6-10 \mathrm{~mm}$ in diameter and with a length of $15 \mathrm{~mm}$. With the help of a stamp a press-fit transplantation of the cylinder, congruently with the surrounding cartilage surface, is created in the defect (Fig. 1). The donor site was filled with remnants of residual cartilage from the defect or it was left open to be filled with fibrocartilaginous tissue within a couple of weeks.

\section{RESULTS}

Eleven out of thirteen patients rated the function of the knee higher after the operation and two patients gave the same score before and after. The mean score before operation was 4.7 and afterwards 7.2. This difference was significant $(p=.007)$. The mean Lysholm-score was 80.9. Six patients scored excellent/good and eight patients scored fair. Twelve out of thirteen patients could return to sports at a medium or high level.

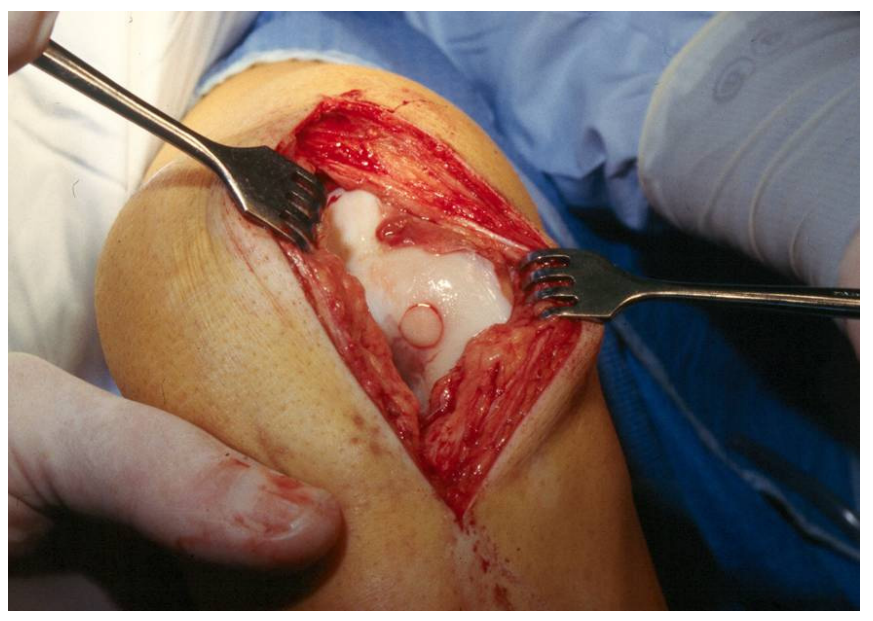

Fig. (1). Donor plug is placed into the cartilage defect in the knee.

The knee examination revealed no abnormal instability or strong restriction of movements. The total mean IKDC score was 58.3. Radiographs did not show an extensive progression of joint space narrowing. The mean improvement in the function score for the two patients who had simultaneously an anterior cruciate ligament reconstruction was 5 in comparison with 2.1 for patients without such a reconstruction. This improvement for patients with simultaneously an ACL-reconstruction was not significant $(\mathrm{p}=.103)$.

Three patients had a second look operation. One patient needed lavage because of a purulent arthritis. Two patients needed a second operation because of sustained complaints caused by an exophyte at the donor site and a remaining cartilage defect.

\section{DISCUSSION}

The most important findings of this study are the objective and subjective improvement in functional outcome of patients treated with the OATS technique with localized articular cartilage defects in the knee.

In our study population, eight patients underwent earlier a (partial) menisectomy or ACL reconstruction. Also three patients had a reconstruction of the ACL during the same procedure as the OATS. It is therefore difficult to distinguish the outcomes of the OATS technique in a knee with more problems. However, patients with a cartilage defect often have additional pathology in the same knee.

In this study, results of the OATS technique are investigated by patient assessment (Lysholm) scores, physical examination and a subjective score using the IKDC scoring system. It would be better to perform a control arthroscopy to view the articular surface and take biopsies or to make MRI's. Because of ethical and financial reasons it was not possible in this study.

A further limitation of our study is the small number of patients and the retrospective nonrandomized uncontrolled study design with short to midterm follow-up. Another restriction of the study is that the patients are scored at 
different postoperative times and it would be better to score at fixed pre- and postoperative moments.

The goal of the treatment of symptomatic articular cartilage defects is to restore the integrity of the joint surface to provide good pain-free function and to prevent eventual progression to serious osteoarthritis. In the literature, various therapeutic options to restore cartilage lesions have been described. One of the basic techniques is lavage with or without mechanical debridement. In this procedure, the inflammatory mediators and the debris are washed out. This joint lavage gives symptomatic improvement in $45 \%$ of the patients and up to $88 \%$ when mechanical debridement was added. The reduction of symptoms is temporary because lavage and debridement alone may not be sufficient to protect from further progression. The underlying pathology is not corrected [3].

Other marrow-stimulation techniques like abrasion, subchondral drilling and making microfractures treat the defect by filling it with fibrocartilaginous repair tissue. These techniques are relatively non-invasive, but fibrocartilaginous tissue is more vulnerable than healthy hyaline cartilage [3]. These therapeutic options do not restore normal hyaline cartilage and have a short-term success rate [2]. After several years, symptoms return because of the progression of osteochondritis which frequently leads to a need for total joint replacements.

Another technique is the autologous chondrocytes transplantation where autologous chondrocytes are obtained from an uninvolved area in the knee and are cultured for two to three weeks. Afterwards they are injected into the area of the defect. The defect is covered with a sutured periosteal flap taken from the proximal tibia. This technique was first described by Brittberg et al. in 1994 [9]. With the autologous chondrocytes transplantation the hyaline cartilage is recovered and several studies show good long term results with success percentages of up to $91 \%$ [4, 5, 9-12]. However the costs are high, and a second operation is necessary.

Several researchers described the advantages of the OATS technique [13-15]. Gudas et al. compared in a randomized controlled trial the outcomes of mosaic-type osteochondral autologous transplantation with the outcomes of microfractures for the treatment of cartilage defects in the knee joints of active athletes [1,2]. This study showed a significant superiority of OATS over microfractures. They found good to excellent results with $96 \%$ of patients in the OATS group compared to $52 \%$ in the microfracture group three years after the operation. Respectively $93 \%$ and $52 \%$ of the athletes could return to sports activities at the pre-injury level at an average of 5 to 6 months [1,2]. Another randomized clinical trial compared the OATS technique with the autologous chondrocyte transplantation [14]. According to the postoperative Lysholm score, the recovery of patients treated with autologous chondrocyte transplantation was slower than the patients treated with the OATS technique. The Meyer and Tegner scores did not show a significant difference in either group after two years. The OATS technique showed better histological results [14]. However Bentley et al. found no significant difference in Cincinnati and Stanmore functional assessment scores between the OATS group and autologous chondrocyte transplantation in a randomized clinical trial after one year [15]. But after one year the International Cartilage Repair Society grading by arthroscopy was significantly better for the autologous chondrocyte transplantation than for patients treated with the OATS-technique. Both randomized clinical trials differed in the following parameters: Size of the defect and allowing weight bearing of the knee directly after the operation or not. In our study, patients with defects larger than $4 \mathrm{~cm}^{2}$ were excluded, and our patients progressed to full weight bearing within 4 to 6 weeks postoperatively instead of directly after the operation. This may be the reason for the comparable results of our study with the good results of Horas et al. [14].

The OATS technique is less viable for cartilage defects larger than $4 \mathrm{~cm}^{2}$ because it is limited by the size of available donor areas. The autologous chondrocyte transplantation seems to be better for larger defects.

\section{CONCLUSION}

Patients with cartilage defects in the knee form an important category in (sports) medicine. Various techniques are described for restoration of full-thickness articular cartilage defects. Resurfacing techniques where the defect is filled with hyaline cartilage, like the OATS technique, are more physiological and durable than fibrocartilagous remodelling treatments. Literature on the treatment of cartilage defects with osteochondral autologous transplantation shows encouraging results. Despite the described limitations of our retrospective nonrandomised study without a control group, the patients in our study population improved after the OATS technique. Although the OATS technique is limited by donor-site availibilty, we consider it to be an appropriate treatment for localized articular cartilage defects and prevent progression of symptoms.

\section{ABBREVIATIONS}

$$
\begin{array}{ll}
\mathrm{ACL} & =\text { Anterior Cruciate Ligament } \\
\mathrm{IKDC} & =\text { International Knee Documentation Committee } \\
\mathrm{KEF} & =\text { Knee Examination Form } \\
\text { OATS } & =\text { Osteochondral Autograft Transplant System } \\
\text { OCD } & =\text { Osteochondritis Dissecans } \\
\text { SKSQ } & =\text { Subjective Knee Score Questionnaire }
\end{array}
$$

\section{REFERENCES}

[1] Gudas R, Stankevičius E, Monastyreckiene E, Pranys D, Kalesinskas RJ. Osteochondral autologous transplantation versus microfracture for the treatment of articular cartilage defects in the knee joint of athletes. Knee Surg Sports Traumatol Arthrosc 2006; 14(9): 834-42.

[2] Gudas R, Kalesinskas R, Kimtys V, et al. A prospective randomized clinical study of mosaic osteochondral autologous transplantation versus microfracture for the treatment of osteochondral defects in the knee joint in young athletes. Arthroscopy 2005; 21(9): 1066-75.

[3] Minas T, Nehrer S. Current concepts in the treatment of articular cartilage defects. Orthopedics 1997; 20(6): 525-38.

[4] Erggelet Ch, Browne JE, Fu F, Mandelbaum BR, Micheli LJ, Mosely JB. Autologous chondrocyte transplantation for treatment of cartilage defects of the knee joint. Clinical results. Zentralbl Chir 2000; 125(6): 516-22.

[5] Steinwachs MR, Erggelet C, Lahm A, Guhlke-Steinwachs U. Klinische und zellbiologische aspekte der autologen chondrozytenimplantation. Methode, indikation und wissenschaftliche ergebnisse. Unfallchirurg 1999; 102(11): 855-60. 
[6] Cameron ML, Briggs KK, Steadman JR. Reproducibility and reliability of the outerbridge classification for grading chondral lesions of the knee arthroscopically. Am J Sports Med 2003; 3: 8386.

[7] Haverkamp D, Sierevelt IN, Breugem JM, Lohuis K, Blankevoort $\mathrm{L}$, Dijk van CN. Translation and validation of the Dutch version of the international knee documentation committee subjective knee form. Am J Sports Med 2006; 34: 1680-84.

[8] Lysholm J, Gillquist J. Evaluation of knee ligament surgery with special emphasis on use of scoring scale. Am J Sports Med 2006; 34: $1680-4$.

[9] Brittberg M, Lindahl A, Nilson A. Treatment of deep cartilage defects in the knee with autologous chondrocyte transplantation. $\mathrm{N}$ Engl J Med 1994; 331(14): 889-95.

[10] Peterson L, Minas T, Brittberg M, Linddahl A. Treatment of osteochondritis dissecans of the knee with autologous chondrocyte transplantation: Results at two to ten years. J Bone Joint Surg Am 2003; 85-A(Suppl 2): 17-24.
[11] Mithöfer K, Peterson L, Mandelbaum BR, Minas T. Articular cartilage repair in soccer players with autologous chondrocyte transplantation: Functional outcome and return to competition. Am J Sports Med 2005; 33(11): 1639-46.

[12] Minas T. Chondrocyte implantation in the repair of chondral lesions of the knee: Economics and quality of life. Am J Orthop 1998; 27(11): 739-44.

[13] Hangody L, Füles P. Autologous osteochondral mosaicplasty for the treatment of full-thickness defects of weight-bearing joints: Ten years of experimental and clinical experience. J Bone Joint Surg Am 2003; 85A; (Suppl 2): 25-32.

[14] Horas U, Pelinkovic D, Herr G, Aigner T, Schnettler R. Autologous chondrocyte implantation and osteochondral cylinder transplantation in cartilage repair of the knee joint. A prospective, comparative trial. J Bone Joint Surg Am 2003; 85-A(2): 185-92.

[15] Bentley G, Biant LC, Carrington RW, et al. A prospective randomised comparison of autologous chondrocyte implantation versus mosaicplasty for osteochondral defects in the knee. J Bone Joint Surg Br 2003; 85(2): 223-30.

(C) Muller et al.; Licensee Bentham Open.

This is an open access article licensed under the terms of the Creative Commons Attribution Non-Commercial License (http://creativecommons.org/licenses/by$\mathrm{nc} / 3.0 /$ ) which permits unrestricted, non-commercial use, distribution and reproduction in any medium, provided the work is properly cited. 